\title{
Despite calls to action, Black scientists remain underrepresented at neuroscience meetings
}

\section{BY PETER HESS}

8 NOVEMBER 2021

\section{Listen to this story: \\ https://www.spectrumnews.org/wp- content/uploads/2021/11/audio-1ebedf51-76b4-4a97-9d5c-215413c284d7-encodings.mp3}

Since the Black Lives Matter protests of 2020, the proportion of presenters at neuroscience conferences who are Black has increased by only 3 percentage points, according to a new analysis.

The study, published Thursday in Nature Neuroscience, compared the fraction of Black presenters at 18 neuroscience conferences that took place from May 2019 to the end of January 2020 with that at 18 meetings that occurred between October 2020 and the end of May 2021.

Only 3 out of 18 conferences during the earlier time period had any Black presenters, and Black scholars made up just 1.2 percent of the total number of speakers. By the end of May 2021, those figures had risen only marginally: 7 out of 18 conferences had Black presenters, who made up 4.2 percent of the total number.

In other words, the median number of Black speakers at neuroscience conferences was zero for both time periods.

"It was about what I expected, unfortunately," says study investigator Lewis Wheaton, associate professor of biological sciences at the Georgia Institute of Technology in Atlanta.

Seeing all those zeros in Wheaton's data was jarring, but not surprising, says Rackeb Tesfaye, a graduate student in Mayada Elsabbagh's lab at McGill University in Montreal, Canada, and co- 


\section{Spectrum | Autism Research News}

https://www.spectrumnews.org

founder of the advocacy group Black in Neuro. The conference data speak to deeper, more systemic issues facing Black researchers, whose work is cited less often in academic literature, who tend to occupy a disproportionately low number of tenured positions and who generally feel less comfortable in academic settings, she says. The data help illuminate the problems and guide discussions, but at some point the neuroscience community needs to commit to making use of the talent and resources that are already available.

As an illustration, Black in Neuro attracted 70 abstract submissions from Black graduate students when it put on its own virtual conference in 2020, after the Society for Neuroscience (SfN) annual meeting was canceled because of the coronavirus pandemic, Tesfaye says.

"If a group of trainees is able to do that, it kind of begs the question: What are some of these committees doing?"

!function()\{"use strict";window.addEventListener("message",(function(e)\{if(void 0 !==e.data["datawrapper-height"])\{var $\mathrm{t=document.querySelectorAll("iframe");for(var} \mathrm{a} \mathrm{in}$ e.data["datawrapper-height"])for(var $r=0 ; r$ 